\title{
Antioxidants in Thai Herb, Vegetable and Fruit Inhibit Hemolysis and Heinz Body Formation in Human Erythrocytes
}

\author{
Warin Sangkitikomol \\ Chulalongkorn University, Center for Excellence in Omics-Nano Medical Technology \\ Development Project, Department of Clinical Chemistry, \\ Faculty of Allied Health Sciences, Bangkok, \\ Thailand
}

\section{Introduction}

Over centuries, Thai traditional herbal beverage and herbal tea have played an important role not only in quenching the thirst but also in providing therapy for common ailments. Recently, ready-to-drink beverages; tea, herbal-tea and fruit juice, are become very popular for Thai people, especially the teenagers. Because of the media presents results of promoting health and preventing many diseases by intake fruit, vegetable and herb which contained high level of antioxidant activities. These plants are rich of antioxidants those are capable to terminate free radical reactions and prevent oxidative damage. Vegetables and fruits are important sources of antioxidant in diet. Scientists have been searching for more than 2 decades to identify the specific ingredients in fruit vegetable and herb that account for their many health promotion benefits. That search points to plant metabolites, many of which are antioxidants, phytochemicals, mixtures of vitamins and fiber content. Thousands experimental studies have examined the role of specifics flavonoids or phenolics in disease prevention(Sen et al., 2010; Virgili \& Marino, 2008). Different phytochemicals in herbal products are safer than synthetic medicine and beneficial in the treatment of diseases caused by free radicals. Multiple biological effects of them have been described, among them; antioxidants, cellular signals, cardioprotective effects, antibiotics, antiinflammation, antiallergic, anticoagulation, antineoplastic, anti-mutagenesis, anti-carcinogenesis (Ames et al.,1993; Lin and Liang, 2000; Ziegler, 1991; Packer and Colman,1999; Halliwell and Gutteridge, 2001; Nakamura, 1997; Cook \& Samma, 1996; Chen and Yen, 1997; Lin \& Liang, 2000).

The major fraction of those plants is nutraceuticals, flavonoids, for neutralizing stress induced free radicals. Flavonoids are effective antioxidants and may protect against several chronic diseases. Flavonoids are divided into six different classes (flavanols, flavanones, flavones, isoflavones, flavonols and anthocyanins) depended on different molecular structure. The flavonol quercetin and the flavone apigenin are found in many fruits and vegetables such as onions, apples, broccoli, and berries. Catechins are main flavonoids in green tea. Cyanidin and anthocyanidins are the pigment of black rice, berries and grapes. Genistein is an isoflavone found in legumes. Hesperitin, Naringenin and Eriodictyol are 
flavanones in citrus fruits. The group of flavanol catechins is derived from green tea, red grapes, red wine and chocolate (Beecher, 2003). The antioxidant activities of phenolics are mainly due to their redox properties which allow them to act as reducing agents, hydrogen donors, and singlet oxygen quenchers. In addition they have a metal chelation potential (Halliwell and Gutteridge, 2001). Flavonoids may suppress LDL oxidation and inflammatory progression in the artery wall (Hertog et al, 1995; Peluso, 2006). It was found that catechins promote many biological functions, including prevention of cardiovascular diseases (Langley-Evans, 2001) and cancer (Kohlmeier et al, 1997; Steinmetz \& Potter, 1991). Polyphenolic phytochemicals such as epigallocatechin from tea, the flavonoids quercetin and genistein from onions and soya, curcumin in curry spice and resveratrol from red grapes are diet constituents with high efficacy in preclinical carcinogenesis of colorectum, breast and prostate (Thomasset et al., 2006; Lambert et al., 2005; Surh, 2003). Low cerebrovascular disease was associated with high intake of the flavonol kaempferol and of the flavonones naringenin and hesperitin and there was a trend of reduction in type 2 diabetes was associated with higher quercetin and myricetin intake (Knekt et al., 2002). The other reported a strong inverse association between the sum of quercetin, myricetin, luteolin, and apigenin intaked and stroke (Keli et al.,1996) and low risk of lung cancer at high flavonoids intake (Knekt et al.,1997). Black rice anthocyanins reduced oxidative damage (Sangkitikomol et al., 2010a), anti-inflammation (Wang et al., 2007), enhanced LDLreceptor (Sangkitikomol et al., 2010b) and promoted cardiovascular health status (Ling et al., 2002; Wang et al., 2007). Aging is the major risk factor for neurodegenerative diseases such as Parkinson's diseases and Alzheimer's. Polyphenolic compounds could affect on cells not only due to their antioxidant activities but also due to their modulation of different pathways including signaling cascades, antiapoptotic processes and the synthesis of the amyloid $\beta$ peptide (Ramassamy, 2006).

CytochromeP450 isoenzymes (CYP450s) are major enzymes in phase I of biotransformation system which involved in the metabolism of various endogenous chemicals such as; fatty acids, steroids, hormones, bile acids, eicosanoids and exogenous chemicals such as; xenobiotics, carcinogens, mutagens and environmental pollutants. A major function of CYP450s is catalyzed the reactions then conversion these nonpolar compounds into polar metabolites which can be conjugated by phase II enzymes. The oxygen activation by CYP450s' catalytic function generate the reactive oxygen species (ROS) (Guengerich, 2008). Flavonoids may be beneficial for health protection by reducing oxidative damage and minimizing toxicity by regulating mRNA CYP450s expression for suitable production or inhibition of CYP450s isoenzymes, thereby maintaining xenobiotic biotransformation balance. Flavonoids from St. John's wort can selectively inhibit CYP1 enzymes may be useful as chemoprotective agents in prostate cancer (Chaudhary \& Willett, 2006). Inhibition of PAH-induced carcinogenesis using cancer chemoprevention; methoxylated flavones and stilbene resveratrol, could effectively inhibit the benzo[ $a]$ pyrene-DNA binding and CYP $1 \mathrm{~A}$ induction which were the early step in molecular levels of cencer prevention (Tsuji \& Walle, 2007). Emerging evidence indicates that transcriptional activation of the antioxidant response element (ARE) plays a crucial role in modulating oxidative stress and providing cytoprotection against prooxidant stimuli (Nguyen et al., 2003). Several chemopreventive agents, such as curcumin, caffeic acid phenethyl ester, rectinoic acid, (-)-epigallocatechin-3gallate and (-)-epicatechin-3-gallate from tea, directed to protect DNA and other important 
cellular molecules by inducing the synthesis of phase II detoxifying genes and antioxidant genes via the Nrf2-ARE signaling pathway. Thereby enhancing those genes transcription and stimulating carcinogen detoxification/inactivation. The Nrf2 (nuclear transcription factor erythroid 2p45 (NF-E2)-related factor 2), is a regulator of genes encoding antioxidants and phase II enzymes such as glutathione S-transferase, NAD(P)H:quinine oxidoreductase 1 , UDP-glucuronosyl-transferase, $\gamma$-glutamate cysteine ligase, and hemeoxygenase- 1 . The Nrf2 is known to mediate detoxification and/or to exert antioxidant functions thereby protecting cells from genotoxic damage (Lee \& Surh, 2005; Zhang \& Gordon, 2004; Hayes \& McMahon, 2001). NF-kB regulated genes such as inducible nitric oxide synthase (iNOS) and cyclooxgenase-2 (COX-2) which are the inflammatory mediators and may promote cancinogenesis (Greten \& Karin, 2004; Surh, 2003). Nrf2 encodes for antioxidant and general cytoprotection genes, while NF- $\mathrm{kB}$ regulates the expression of proinflammatory genes. A variety of antiinflammatory or anticarcinogenic phytochemicals suppress NF- $\mathrm{kB}$ signalling and activate the Nrf2-ARE pathway (Bellezza et al., 2010)

There is no evidence that different types of tea, herbal tea, fruit and vegetable currently available in the markets of Thailand have any significant antioxidant contents. The aim of this study was to carry out a survey of relative levels of total antioxidant activities by TEAC assay (Re et al., 1999) with reference to their total phenolic contents (Singleton et al., 1999). In order to find the natural sources of antioxidants, thereafter some of them were selected to study the inhibition effect on hemolysis (Sangkitikomol et al.,2010a) as a marker of lipid peroxidation, induced by 2, 2'-azobis (2-amidinopropane) hydrochloride and the inhibition effect on Heinz body formation, as a marker of protein oxidation, induced by $\mathrm{N}$ acethylphenylhydrazine (Sangkitikomol et al., 2001; 2010a) by using normal fresh human red blood cells.

\section{Measuring antioxidant activities of tea, herbal tea, fruit and vegetable}

\subsection{Chemicals}

ABTS [2,2'-azinobis-(3-ethylbenzothiazoline-6-sulphonic acid)] diammonium salt, TPTZ (2,4,6-tripyridyl-s-triazine), gallic acid, Trolox(6-hydroxy-2,5,7,8-tetramethylchroman-2carboxylic acid), AAPH[2,2'-azobis(2 amidinopropane) dihydrochloride], Folin Ciocalteu's phenol reagent and APHZ (N-acethylphenylhydrazine) were purchased from SigmaAldrich, St. Louis, MO,USA. All other basic reagents were of analytical grade.

\subsection{Preparation of plant extracts}

Different types of Thai plants were purchased from Thai-herb shops, health food shops, and local markets. These samples were crushed into small pieces and some were ready-made, they were 152 samples of fruit, vegetable and herb, 33 brands of tea. A hundred milligram of dry material was extracted with $2 \times 10 \mathrm{ml}$ of solvent using ultrasonic bath for 5 minutes sonication, centrifuged at $3000 \mathrm{rpm}$ for 10 minutes and the combined extracts were kept in deep freezer at $-80^{\circ} \mathrm{C}$ until used. Using 2 kinds of solvent for extraction, one is $80 \%$ aqueous methanol and the other is deionized reverse osmosis water. The plant water-extracts were used to determine lipid and protein oxidation in human red blood cells, and the other were used to analyze the antioxidant activity. Fourteen samples of ready-to-drink beverages were purchased from supper markets and directly used for analysis. 


\subsection{Preparation of fresh whole blood}

Human blood samples were obtained from The National Blood Centre, Thai Red Cross Society, Bangkok, Thailand. Fresh blood was collected in heparinised tubes and centrifuged at $252 \times \mathrm{g}$ for $3 \mathrm{~min}$. Plasma was carefully removed by aspiration in order to obtain a hematocrit of approximately $50 \%$ (packed red blood cells; RBCs) for hemolysis test and Heinz body formation. The blood was stored at $4^{\circ} \mathrm{C}$ and used within $3 \mathrm{~h}$.

\subsection{Determination of antioxidants in plant extracts}

The total antioxidant determination and total phenolic contents were performed on the Shimadzu spectrophotometer model UV160A (Tokyo, Japan). All determinations were carried out at least three times of the standards and samples. And the correlation analysis had been done by using program Microsolf Excel 2007.

\subsubsection{Trolox Equivalent Antioxidant Capacity (TEAC) Assay}

Total antioxidant activity is measured by TEAC assay of Re et al. (1999). It is a screening of lipophilic and hydrophilic antioxidants activity by decolorization assay. The radical monocation of 2,2'-azinobis-(3-ethylbenzothiazoline-6-sulfonic acid) (ABTS•) is generated by oxidation of ABTS with potassium persulfate. Then the radical ABTS $\bullet$ is reduced in the presence of hydrogen-donating antioxidants from the plant extracts. The percentage inhibition of absorbance at $734 \mathrm{~nm}$ is calculated and plotted as a function of concentration of antioxidants and of Trolox for the standards. The results were expressed as Trolox equivalent in $\mathrm{mM}$ per kilogram of dry weight (TE $\mathrm{mM} / \mathrm{kg} . \mathrm{dw})$.

\subsubsection{Folin cioculteau phenol assay (Folin assay)}

Modified Folin Cioculteau Phenol assay (Singleton et al., 1999) is used to determine reducing properties of phenolics contents. Briefly, $500 \mu \mathrm{l}$ of samples or standards was mixed with $500 \mu \mathrm{l}$ of $10 \%$ Folin reagent, let stand for $20 \mathrm{~min}$, added $10 \mathrm{mM} \mathrm{Na}_{2} \mathrm{CO}_{3} 350 \mu \mathrm{l}$, let stand for $20 \mathrm{~min}$ for the solution turned blue color and then measured the absorbance at $750 \mathrm{~nm}$ by using gallic acid as the standards. The results were expressed as Gallic acid equivalent (GE) $\mathrm{mM} / \mathrm{kg} . \mathrm{dw}$.

\subsubsection{Determination of AAPH-Induced oxidative damage of erythrocyte membrane}

The erythrocyte susceptibility to oxidative destruction was evaluated in vitro by subjecting the cells to oxidative stress. Since a peroxyl radical initiator, AAPH, has been proved to cause an oxidation of cell membrane proteins and lipids resulting in lysis of red blood cells (RBCs), oxidative hemolysis induced by AAPH (Sangkitikomol et al.,2010a) was used as a tool in the present study. Briefly, positive control was added $0.100 \mathrm{ml}$ of fresh whole blood into pre-incubated medium [1.00 $\mathrm{ml}$ of phosphate buffer saline (PBS) $\mathrm{pH}$ 7.4], then incubated at $37{ }^{\circ} \mathrm{C}$ for $5 \mathrm{~min}$. One $\mathrm{ml}$ of PBS solution with $200 \mathrm{mM}$ AAPH was added to the whole blood suspension. The reaction mixture was shaken gently at $37{ }^{\circ} \mathrm{C}$ under aerobic condition for three and a half hours. Reaction mixture $(0.100 \mathrm{ml})$ was withdrawn to $1.5 \mathrm{ml}$ of ice cold PBS at 120, 150, 180 and 210 minutes after added AAPH and centrifuged at $1006 \times \mathrm{g}$ for $10 \mathrm{~min}$. The extent of hemolysis was determined by measuring the absorbance of 
hemolysate at $540 \mathrm{~nm}$. Negative control was done the same as positive control without AAPH presented. And the plant extracts was done the same as positive control excepted only using the extract $0.5 \mathrm{ml}$ mixed with $0.5 \mathrm{ml}$ of double strength of PBS in stead of $1.00 \mathrm{ml}$ pre-incubated medium. Similarly, $0.100 \mathrm{ml}$ of reaction mixture of negative control was treated with $1.5 \mathrm{ml}$ of distilled water to yield complete hemolysis. Percentage hemolysis was calculated according to the equation. \% hemolysis = absorbance of the sample aliquot divided by absorbance of the complete hemolysis $\times 100$. Data was represented as the time required to achieve $50 \%$ hemolysis ( $\left.\mathrm{T}_{50} \mathrm{~min}\right)$.

\subsubsection{Determination of APHZ-Induced Heinz body formation in cytosol of erythrocytes}

APHZ was used as a free radical initiator inside RBCs to oxidize proteins mostly hemoglobin based on the modification of protocol described previously (Sangkitikomol et al. 2001). Using Heinz body formation was a marker of protein oxidation for testing the antioxidants properties of plant extracts. Briefly, positive control was added $0.1 \mathrm{ml}$ of fresh whole blood into $2.0 \mathrm{ml}$ of reaction medium [contained $1.0 \mathrm{~g} / \mathrm{L}$ of APHZ, $2.0 \mathrm{~g} / \mathrm{L}$ of glucose in phosphate buffer $\left(1.3\right.$ parts of $1 / 15 \mathrm{M}^{\circ} \mathrm{KH}_{2} \mathrm{PO}_{4}$ and 8.7 parts of $1 / 15 \mathrm{M}$ of $\mathrm{Na}_{2} \mathrm{HPO}_{4}, \mathrm{pH}$ 7.4)] and incubated for 2 hours at $37{ }^{\circ} \mathrm{C}$ under aerobic condition. Heinz bodies are precipitates of oxidized or denatured hemoglobin that adhere strongly to the red blood cell membrane. Negative control was done without APHZ added. And the plant extracts were done the same as positive control excepted only using the plant extract $1.0 \mathrm{ml}$ mixed with $1.0 \mathrm{ml}$ of double strength of reaction medium. Staining Heinz bodies in RBC with 3 drops of crystal violet solution ( $10 \mathrm{~g} / \mathrm{L}$ in $0.73 \%$ of normal saline) and 3 drops of RBC from reaction medium for 5 minutes at room temperature. Made blood smear on glass slides and counted RBC with Heinz body inside/1,000 of RBC using light microscope. The results were reported in \% inhibition of Heinz body formation.

\subsubsection{Results}

Antioxidant activities of 152 herbs and 33 brands of tea using TEAC assay varied considerably with the types of plants and the types of tea.The range of antioxidant activities and total phenolic contents were several hundred-fold. The plants which are very good sources of antioxidants are Quercus infectoria, Areca catechu Linn., Terminalia spp., Phyllanthus emblica Linn., Punica granutum Linn., Eugenia caryophyllus (Table 1) and tea (Camellia sinensis) (Table 2). Correlation analysis (R) between the antioxidant activity measurements of plantextracts and tea-extracts with the measured total phenol concentration were calculated to be 0.988 and 0.902 , respectively (Figure 1-2). Antioxidant activities varied considerably with the types of tea (Table 2). In the TEAC assay tea-extracts with the highest to the lowest activity were green tea, oolong tea and black tea, respectively. The range of antioxidant activity was 15.6-fold for the TEAC analysis and 6.6-fold for Folin assay. Fourteen samples of ready-todrink beverages were found small amount of polyphenolics and antioxidant contents (Table 3). Selected 30 samples with high level of total antioxidant activities, within the range 141$9490 \mathrm{mM} \mathrm{TE} / \mathrm{kg}$.dw., were used to study the inhibition effect of lipid peroxidation and protein oxidation. The results showed that 13 out of 30 samples showed inhibition effect on Heinz body formation and the other had no effect may be caused by antioxidant contents in the extracts was not enough to inhibit Heinz body formation inside red blood cells. All 
samples showed prolong $\mathrm{T}_{50}$ min. of hemolysis assay, and most of them had very strong inhibition effect which showed the $T_{50} \mathrm{~min}$. were longer than 3 and a half hours. Time required to get $\mathrm{T}_{50} \mathrm{~min}$. of control positive was between 120-150 min. (Table 4).

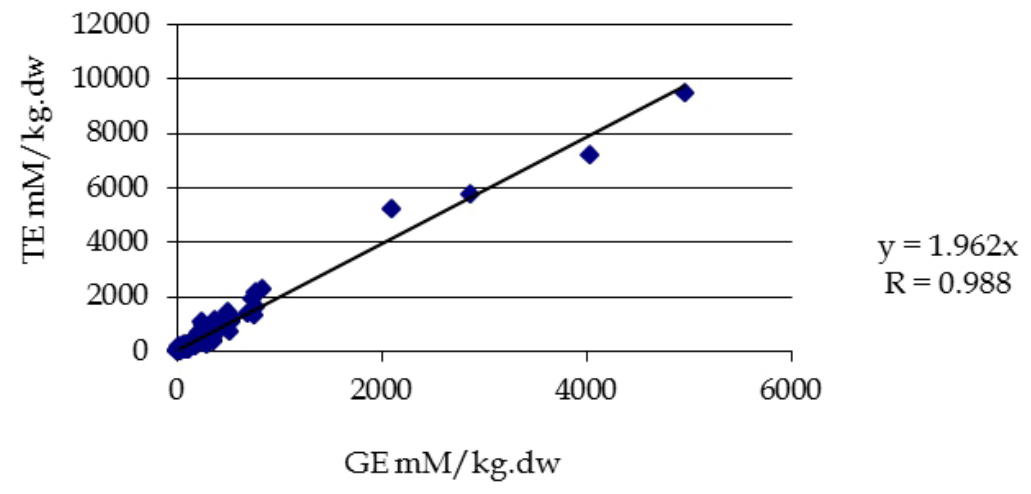

Fig. 1. Correlation between polyphenolics using Folin assay with total antioxidant activities using Trolox equivalent antioxidant capacity in 152 plant-extracts. Both assays are expressed in dry weight.

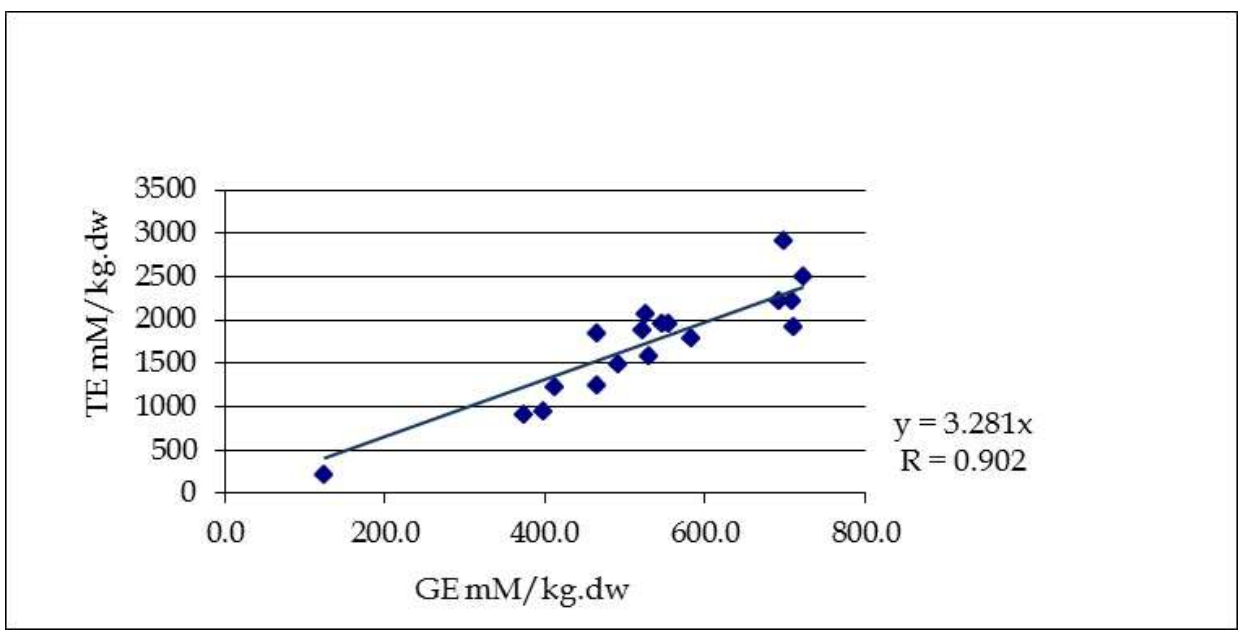

Fig. 2. Correlation between 33 polyphenolics using Folin assay with total antioxidant activities using Trolox equivalent antioxidant capacity in 33 tea-extracts. Both assays are expressed in dry weight. 
Antioxidants in Thai Herb, Vegetable and

\begin{tabular}{|c|c|c|c|}
\hline № & Scientific Name of plants & $\begin{array}{c}\text { Folin Assay } \\
\text { GE mM/kg.dw. }\end{array}$ & $\begin{array}{c}\text { TEAC Assay } \\
\text { TE mM/kg.dw. }\end{array}$ \\
\hline 1 & Abroma augusta Linn. & $63+3$ & $40 \pm 3$ \\
\hline 2 & Acacia catechu Willd. & $4038 \pm 106$ & $7183 \pm 204$ \\
\hline 3 & Acacia rugata Merr. & $94+10$ & $52+1$ \\
\hline 4 & Adhatoda vasica (L.) Nees & $47 \pm 3$ & $56 \pm 10$ \\
\hline 5 & Aiiium ascalonicum Linn. & $221+30$ & $357+20$ \\
\hline 6 & Albizia myriophylla Benth. & $61+8$ & $144+7$ \\
\hline 7 & Allium tuberosum Roxb. & $36 \pm 4$ & $80.8 \pm 3.5$ \\
\hline 8 & Amomum cadamomum & $5 \pm 1$ & $12.0 \pm 1.0$ \\
\hline 9 & Anethum graveolens Linn. & $47 \pm 4$ & $55 \pm 4$ \\
\hline 10 & Angelina sinensis & $25+2$ & $46+4$ \\
\hline 11 & Areca catechu Linn. & $2876 \pm 68$ & $5771 \pm 46$ \\
\hline 12 & Artimisia pallens Wall. Ex Bess. & $35 \pm 5$ & $44 \pm 5$ \\
\hline 13 & Artocapus heterophyllus Lamk. & $116+10$ & $29+4$ \\
\hline 14 & Artocarpus lakoocha Roxb. & $744 \pm 32$ & $1923 \pm 201$ \\
\hline 15 & Atractylodes lyrata Sieb.et Zucc. & $19+3$ & $31+6$ \\
\hline 16 & Azodirachta indica A Juss. (stem) & $237 \pm 10$ & $467 \pm 39$ \\
\hline 17 & Azodirachta indica A Juss. (flower) & $53+2$ & $99+8$ \\
\hline 18 & Azodirachta indica A Juss. (leaf) & $188+10$ & $322+35$ \\
\hline 19 & Baliospermum montanum Willd. & $38 \pm 2$ & $64 \pm 6$ \\
\hline 20 & Baliospermum montanum Willd. & $157+4$ & $212+15$ \\
\hline 21 & Bambusa spp. & $42 \pm 3$ & $74 \pm 10$ \\
\hline 22 & Boesenbergia pandurate Holtt. & $107+6$ & $221+15$ \\
\hline 23 & Bougainvillea spectabilis Willd. & $157 \pm 10$ & $218+12$ \\
\hline 24 & Brassica camprestris L ssp. & $59 \pm 2$ & $144 \pm 11$ \\
\hline 25 & Bridelia siamensis Craib. & $98+8$ & $129+10$ \\
\hline 26 & Caesalpinia sappan Linn. & $520 \pm 15$ & $1292 \pm 102$ \\
\hline 27 & Cantharanthus roseus (flower) & $95+10$ & $134+12$ \\
\hline 28 & Cantharanthus roseus (leaf) & $107 \pm 7$ & $151+23$ \\
\hline 29 & Carica papaya Linn. (leaf) & $47 \pm 2$ & $90 \pm 3$ \\
\hline 30 & Carum carvi Linn. & $53+5$ & $66 \pm 10$ \\
\hline 31 & Casicum frutecens Linn. (fruit) & $58+3$ & $89 \pm 4$ \\
\hline 32 & Casicum frutecens Linn. (seed) & $40 \pm 5$ & $15+3$ \\
\hline 33 & Cassia sophera Linn. & $59 \pm 7$ & $88 \pm 7$ \\
\hline 34 & Cinnamomum cassia & $47 \pm 4$ & $38 \pm 6$ \\
\hline 35 & Cinnamomum zeylanicum Linn. & $219 \pm 22$ & $426 \pm 31$ \\
\hline 36 & Citrus hystrix DC. (leaf) & $56 \pm 10$ & $88 \pm 6$ \\
\hline 37 & Citrus hystrix DC. (peel) & $90+9$ & $254+29$ \\
\hline 38 & Citrus medica Linn. Var. & $53 \pm 6$ & $102 \pm 10$ \\
\hline 39 & Cladogynos orientalis Zipp. Ex Span & $27 \pm 3$ & $32+5$ \\
\hline 40 & Combretum extensum & $61 \pm 7$ & $158+15$ \\
\hline 41 & Combretum quadrangulare Kurz. & $164 \pm 10$ & $399 \pm 29$ \\
\hline 42 & Conioselinum univitatum & $49+8$ & $73+10$ \\
\hline 43 & Connarus ferugineus & $417 \pm 31$ & $767 \pm 60$ \\
\hline 44 & Cuminumc cyminum Linn. & $60 \pm 5$ & $94+10$ \\
\hline 45 & Curcuma longa Linn. & $352 \pm 20$ & $395 \pm 41$ \\
\hline
\end{tabular}




\begin{tabular}{|c|c|c|c|}
\hline No & Scientific Name of plants & $\begin{array}{c}\text { Folin Assay } \\
\text { GE mM/kg.dw. }\end{array}$ & $\begin{array}{c}\text { TEAC Assay } \\
\text { TE mM/kg.dw. }\end{array}$ \\
\hline 46 & Cymbopogon citratus stapf. & $50 \pm 4$ & $64+5$ \\
\hline 47 & Cyperus rotundus Linn. & $22 \pm 1$ & $189 \pm 15$ \\
\hline 48 & Diospyros decandra Lour. & $33+4$ & $66+9$ \\
\hline 49 & Diosyrros mollis Griff & $38 \pm 2$ & $49 \pm 4$ \\
\hline 50 & Dracaena loureiri Gagnep. & $169+9$ & $292+27$ \\
\hline 51 & Emita sonchifolia DC. & $32 \pm 3$ & $49 \pm 7$ \\
\hline 52 & Erythrina suberosa Roxb. & $105 \pm 9$ & $165 \pm 20$ \\
\hline 53 & Eugenia caryophyllus & $687 \pm 15$ & $1400+302$ \\
\hline 54 & Eurycoma longifolia Jack & $23.4 \pm 1.6$ & $34.5 \pm 4.1$ \\
\hline 55 & Garcinia mangostana (peel) & $243+12$ & $1095+206$ \\
\hline 56 & Glycyrrhiza glabra Linn. & $295 \pm 20$ & $242 \pm 30$ \\
\hline 57 & Hamisonia perforata (Lour.) Merr. & $405 \pm 31$ & $887 \pm 41$ \\
\hline 58 & Heracleumsiamnicum Craib. & $42+4$ & $55+5$ \\
\hline 59 & Hibiscus abelmoschus Linn. & $22 \pm 2$ & $30 \pm 2$ \\
\hline 60 & Illicium verum Hook & $100+9$ & $171+16$ \\
\hline 61 & Impormoca reptans Poir. & $377 \pm 25$ & $751 \pm 32$ \\
\hline 62 & Ixora cibdela craib & $84+7$ & $134+10$ \\
\hline 63 & Jatropha gossypifolia Linn. & $41+3$ & $69 \pm 6$ \\
\hline 64 & Jusminum sambac Ait. & $49 \pm 3$ & $71 \pm 9$ \\
\hline 65 & Leucaena leucocephala de Wit & $143+10$ & $316+25$ \\
\hline 66 & Levisticum officinale Koch & $52+3$ & $64+5$ \\
\hline 67 & Lycium chinensis & $62+9$ & $66+8$ \\
\hline 68 & Mammea siamensis & $133 \pm 15$ & $291 \pm 25$ \\
\hline 69 & Mangifera indica Linn. (leaf) & $539 \pm 32$ & $1118+90$ \\
\hline 70 & Mesua ferrea Linn. & $222+38$ & $357 \pm 30$ \\
\hline 71 & Millingtonia hortensis Linn. & $100 \pm 15$ & $133 \pm 12$ \\
\hline 72 & Mimusops elengi Linn. & $52+9$ & $114+11$ \\
\hline 73 & Molindia citrifolia Linn. (leaf) & $41 \pm 7$ & $79 \pm 9$ \\
\hline 74 & Momordica charantia Linn. & $28 \pm 3$ & $29 \pm 3$ \\
\hline 75 & Murdandia lorifomis (Hassk.) & $36 \pm 3$ & $56 \pm 4$ \\
\hline 76 & Musa sapientum Linn. (leaf) & $28 \pm 2$ & $38 \pm 4$ \\
\hline 77 & Myristica fragrans Houtt. & $77 \pm 6$ & $247 \pm 15$ \\
\hline 78 & Myristica fragrans Houtt. & $8 \pm 1$ & $21 \pm 4$ \\
\hline 79 & Nelumbo Mucifera Gaertn (flower) & $500 \pm 3$ & $1014 \pm 101$ \\
\hline 80 & Nelumbo Mucifera Gaertn (leaf) & $308+24$ & $714 \pm 35$ \\
\hline 81 & Nicotiana tabacum & $97 \pm 12$ & $106 \pm 14$ \\
\hline 82 & Nigella sativa Linn. & $83+5$ & $118+11$ \\
\hline 83 & Ocimum basilicum & $56 \pm 4$ & $76 \pm 8$ \\
\hline 84 & Ocimum sanctum Linn. (flower) & $95+7$ & $130+10$ \\
\hline 85 & Ocimum sanctum Linn. (leaf) & $53+4$ & $64+6$ \\
\hline 86 & Pandanus odoratissimus Linn. & $29 \pm 3$ & $36 \pm 4$ \\
\hline 87 & Panicum repens Linn. & $21+4$ & $21+2$ \\
\hline 88 & Petroselium crispum (Mill.) & $107 \pm 10$ & $200 \pm 14$ \\
\hline 89 & Pimpinella anisum Linn. & $59 \pm 5$ & $107 \pm 10$ \\
\hline 90 & Pinus mercukusii Jungh \& de Vriese & $65 \pm 5$ & $83 \pm 7$ \\
\hline
\end{tabular}


Antioxidants in Thai Herb, Vegetable and

\begin{tabular}{|c|c|c|c|}
\hline No & Scientific Name of plants & $\begin{array}{c}\text { Folin Assay } \\
\text { GE mM/kg.dw. }\end{array}$ & $\begin{array}{c}\text { TEAC Assay } \\
\text { TE mM/kg.dw. }\end{array}$ \\
\hline 91 & Piper aurantiacum Mig & $75 \pm 10$ & $108+12$ \\
\hline 92 & Piper betle Linn. & $360 \pm 15$ & $375 \pm 20$ \\
\hline 93 & Piper chaba Hunt. & $31+3$ & $37 \pm 4$ \\
\hline 94 & Piper nigrum Linn(Black pepper) & $24 \pm 4$ & $34 \pm 4$ \\
\hline 95 & Piper sarmentosum Roxb. & $48+4$ & $71+7$ \\
\hline 96 & Pithecellobium tenue Craib & $507 \pm 32$ & $1433 \pm 102$ \\
\hline 97 & Plantago ovata Forskal P. & $17 \pm 2$ & $22 \pm 2$ \\
\hline 98 & Plumbago indica Linn. & $66+6$ & $100+11$ \\
\hline 99 & Plumeria alba Linn. & $21 \pm 3$ & $35 \pm 4$ \\
\hline 100 & Polygonium odoratum Lour. & $168+12$ & $380 \pm 20$ \\
\hline 101 & Psidium guajava & $376 \pm 29$ & $1121 \pm 100$ \\
\hline 102 & Punica granutum Linn. & $778 \pm 60$ & $2157 \pm 180$ \\
\hline 103 & Quercus infectoria & $4962+202$ & $9490+390$ \\
\hline 104 & Rosa domescena Mill. & $428 \pm 24$ & $1035 \pm 95$ \\
\hline 105 & Sidarhombifolia Linn. & $97+18$ & $113+11$ \\
\hline 106 & Smilax corbulalia Kunta & $752+80$ & $1308+99$ \\
\hline 107 & Smilax micro-china T. Koyama & $162+11$ & $239+16$ \\
\hline 108 & Spilanthes acmella Linn. & $44+3$ & $71 \pm 8$ \\
\hline 109 & Spilanthes acmella Linn. & $39 \pm 5$ & $73 \pm 9$ \\
\hline 110 & Stemona toberosa Lour. & $14+2$ & $26 \pm 2$ \\
\hline 111 & Streblus asper Lour. & $16 \pm 1$ & $20 \pm 2$ \\
\hline 112 & Strychnos lucida R. Br. & $81+3$ & $91+8$ \\
\hline 113 & Syzygium gratum & $227 \pm 27$ & $718 \pm 66$ \\
\hline 114 & Tamaridus indica Linn. & $57 \pm 6$ & $104 \pm 11$ \\
\hline 115 & Tamarindus indica Linn. & $255+20$ & $932+46$ \\
\hline 116 & Terminalia chebula Retz. & $772 \pm 65$ & $1640 \pm 112$ \\
\hline 117 & Terminalia spp. & $2100 \pm 100$ & $5216+150$ \\
\hline 118 & Tiliacora triandra Diels & $83+8$ & $86+10$ \\
\hline 119 & Tinospora cordifolia Miers & $65 \pm 8$ & $77 \pm 6$ \\
\hline 120 & Urceola minutiflora Pierre & $172 \pm 10$ & $283 \pm 18$ \\
\hline 121 & Xylinbaria minutiflora Pierre & $539 \pm 50$ & $1050 \pm 97$ \\
\hline 122 & Zingiber cassumunar Roxb. & $97+11$ & $113+11$ \\
\hline 123 & Ziziphus mauritiana & $74 \pm 7$ & $29 \pm 3$ \\
\hline 124 & Andrographis paniculata Nee & $140 \pm 9$ & $164 \pm 10$ \\
\hline 125 & Bulbostylis barbata Clarke & $116 \pm 11$ & $119 \pm 10$ \\
\hline 126 & Carcinia atroviridis Griff. & $22 \pm 2$ & $34 \pm 3$ \\
\hline 127 & Carthamus tinctorius Linn & $142+10$ & $186+10$ \\
\hline 128 & Cassia alata Linn. & $124 \pm 10$ & $208 \pm 14$ \\
\hline 129 & Cassia angustifilia vohl. & $84+9$ & $139+11$ \\
\hline 130 & Cassia tora Linn. & $111 \pm 10$ & $203 \pm 18$ \\
\hline 131 & Centella asiatica Urban & $78 \pm 6$ & $136 \pm 10$ \\
\hline 132 & Chrysanthenum indicum & $96+8$ & $114+10$ \\
\hline 133 & Clitorea ternatea Linn. & $86 \pm 6$ & $156 \pm 10$ \\
\hline 134 & Cymbopogon citratus stapf. & $50+4$ & $64+6$ \\
\hline 135 & Ganoderma lucidum (sample 1) & $21 \pm 2$ & $29 \pm 2$ \\
\hline
\end{tabular}




\begin{tabular}{|l|l|c|c|}
\hline No & \multicolumn{1}{|c|}{ Scientific Name of plants } & $\begin{array}{c}\text { Folin Assay } \\
\text { GE mM/kg.dw. }\end{array}$ & $\begin{array}{c}\text { TEAC Assay } \\
\text { TE mM/kg.dw. }\end{array}$ \\
\hline 136 & Gonoderma lucidum (sample 2) & $19 \pm 2$ & $57 \pm 3$ \\
\hline 137 & Ginkgo biloba & $216 \pm 11$ & $652 \pm 40$ \\
\hline 138 & Mimosa pudica Linn. & $123 \pm 10$ & $193 \pm 19$ \\
\hline 139 & Morus alba Linn. (sample 1) & $56 \pm 4$ & $126 \pm 9$ \\
\hline 140 & Morus alba Linn.(sample 2) & $119 \pm 10$ & $139 \pm 10$ \\
\hline 141 & Nelumbo Mucifera Gaertn (pollen) & $143 \pm 12$ & $290 \pm 18$ \\
\hline 142 & Oroxylum indicum Vent. & $511 \pm 20$ & $710 \pm 32$ \\
\hline 143 & Pandanus amaryllifolius Roxb & $89 \pm 9$ & $141 \pm 11$ \\
\hline 144 & Phyllanthus emblica Linn. & $841 \pm 40$ & $2288 \pm 91$ \\
\hline 145 & Phyllanthus urinaria Linn. & $219 \pm 29$ & $330 \pm 25$ \\
\hline 146 & Rhinacanthus nasutus Kurz Sn. & $16 \pm 1$ & $41 \pm 3$ \\
\hline 147 & Schefflera leucantha (sample 1) & $38 \pm 4$ & $88 \pm 9$ \\
\hline 148 & Schefflera leucantha(sample 2) & $57 \pm 4$ & $82 \pm 6$ \\
\hline 149 & Thunbergia iaurifolia Linn. & $189 \pm 12$ & $190 \pm 11$ \\
\hline 150 & Tinospara erispa Miers & $20 \pm 2$ & $31 \pm 4$ \\
\hline 151 & Zingiber officenale Roscoe & $37 \pm 11$ & $220 \pm 19$ \\
\hline 152 & Zingiber officinale Roscoe (young) & $37 \pm 3$ & $60 \pm 4$ \\
\hline
\end{tabular}

All determinations were carried out at least three times .

*Polyphenolics are expressed as the mean \pm SD in $\mathrm{mM}$ of catechin equivalents per kilogram of dry weights.

${ }^{* *}$ Total antioxidant activities are expressed as the mean \pm SD in $\mathrm{mM}$ of Trolox equivalents per kilogram of dry weights.

Table 1. The polyphenolic contents and total antioxidant activities of 152 plant-extracts using Folin assay and TEAC assay, respectively.

\begin{tabular}{|l|c|c|}
\hline \multicolumn{1}{|c|}{ Type of Tea } & $\begin{array}{c}\text { Folin Assay } \\
\text { GE mM/kg.dw }\end{array}$ & $\begin{array}{c}\text { TEAC Assay } \\
\text { TE mM/kg.dw. }\end{array}$ \\
\hline Green tea (brand 1) & $818 \pm 66$ & $3307 \pm 112$ \\
\hline Green tea (brand 2) & $807 \pm 42$ & $2740 \pm 96$ \\
\hline Green tea (brand 3) & $752 \pm 52$ & $2541 \pm 69$ \\
\hline Green tea (brand 4) & $697 \pm 37$ & $2452 \pm 100$ \\
\hline Green tea (brand 5) & $633 \pm 26$ & $2252 \pm 56$ \\
\hline Green tea (brand 6) & $636 \pm 46$ & $2369 \pm 78$ \\
\hline Green tea (brand 7) & $622 \pm 41$ & $2836 \pm 82$ \\
\hline Green tea (brand 8) & $698+43$ & $1704 \pm 40$ \\
\hline Green tea (brand 9) & $506 \pm 40$ & $1901 \pm 70$ \\
\hline Green tea (brand 10) & $563 \pm 52$ & $1800 \pm 36$ \\
\hline Green tea (brand11) & $466 \pm 12$ & $1650 \pm 45$ \\
\hline Green tea (brand 12) & $444 \pm 16$ & $1236 \pm 56$ \\
\hline Green tea (brand 13) & $438 \pm 28$ & $1459 \pm 55$ \\
\hline Green tea (brand 14) & $422 \pm 25$ & $2500 \pm 85$ \\
\hline Oolong (brand 1) & $722 \pm 43$ & $1926 \pm 38$ \\
\hline Oolong (brand 2) & $711 \pm 44$ & $2214 \pm 83$ \\
\hline Oolong (brand 3) & $709 \pm 28$ & $2906 \pm 97$ \\
\hline Oolong (brand 4) & $698 \pm 17$ & \pm 18 \\
\hline
\end{tabular}




\begin{tabular}{|l|c|c|}
\hline \multicolumn{1}{|c|}{ Type of Tea } & $\begin{array}{c}\text { Folin Assay } \\
\text { GE mM/kg.dw }\end{array}$ & $\begin{array}{c}\text { TEAC Assay } \\
\text { TE mM/kg.dw. }\end{array}$ \\
\hline Oolong (brand 5) & $693 \pm 36$ & $2222 \pm 80$ \\
\hline Oolong (brand 6) & $600 \pm 22$ & $1994 \pm 71$ \\
\hline Oolong (brand 7) & $583 \pm 22$ & $1793 \pm 38$ \\
\hline Oolong (brand 8) & $526 \pm 29$ & $2062 \pm 71$ \\
\hline Oolong (brand 9) & $555 \pm 26$ & $1963 \pm 24$ \\
\hline Oolong (brand 10) & $547 \pm 35$ & $1963 \pm 67$ \\
\hline Oolong (brand 11) & $523 \pm 18$ & $1878 \pm 41$ \\
\hline Oolong (brand 12) & $464 \pm 30$ & $1840 \pm 31$ \\
\hline Oolong (brand 13) & $412+20$ & $1222 \pm 38$ \\
\hline Oolong (brand 14) & $464 \pm 19$ & $1237 \pm 43$ \\
\hline Black tea (brand 1) & $530 \pm 21$ & $1578 \pm 38$ \\
\hline Black tea (brand 2) & $491 \pm 20$ & $1489 \pm 34$ \\
\hline Black tea (brand 3) & $398 \pm 30$ & $942 \pm 18$ \\
\hline Black tea (brand4) & $374 \pm 21$ & $904 \pm 26$ \\
\hline Black tea (brand 5) & $125 \pm 34$ & $212 \pm 6$ \\
\hline
\end{tabular}

All determinations were carried out at least three times .

*Polyphenolics are expressed as the mean \pm SD in $\mathrm{mM}$ of catechin equivalents per kilogram dry weight.

**Total antioxidant activities are expressed as the mean \pm SD in $\mathrm{mM}$ of Trolox equivalents per kilogram dry weight.

Table 2. The polyphenolic contents and total antioxidant activities of 33 tea-extracts using Folin assay and TEAC assay, respectively.

\begin{tabular}{|ll|c|c|}
\hline \multicolumn{1}{|c|}{ Kind of beverages } & $\begin{array}{c}\text { Folin Assay* } \\
\text { GE } \mu \mathrm{M} / \mathrm{L}\end{array}$ & $\begin{array}{c}\text { TEAC Assay** } \\
\text { TE } \mu \mathrm{M} / \mathrm{L}\end{array}$ \\
\hline 1. & Green tea(sample1) & $7,800 \pm 500$ & $11,400 \pm 1,000$ \\
\hline 2. & Green tea(sample2) & $4,200 \pm 200$ & $9,000 \pm 600$ \\
\hline 3. $\quad$ Green tea(sample3) & $5,400 \pm 300$ & $8,900 \pm 700$ \\
\hline $4 . \quad$ Green tea(sample4) & $3,500 \pm 100$ & $5,000 \pm 300$ \\
\hline $5 . \quad$ Grape juice (sample1) & $8,400 \pm 300$ & $12,200 \pm 400$ \\
\hline 6. & Grape juice (sample2) & $3,500 \pm 100$ & $4,600 \pm 200$ \\
\hline 7. & Guava juice & $4,200 \pm 200$ & $4,200 \pm 100$ \\
\hline 8. & Carrot mixed fruit juice & $3,400 \pm 100$ & $4,200 \pm 300$ \\
\hline 9. & Passion fruit tea & $2,800 \pm 100$ & $3,600 \pm 400$ \\
\hline 10. & Pine apple juice(sample1) & $700 \pm 200$ & $2,700 \pm 200$ \\
\hline 11. & Pine apple juice(sample2) & $1,000 \pm 100$ & $2,400 \pm 100$ \\
\hline 12. & Orange juice & $3,000 \pm 100$ & $2,400 \pm 200$ \\
\hline 13. & Apple juice & $1,900 \pm 100$ & $2,000 \pm 100$ \\
\hline 14. & Litchi juice & $600 \pm 100$ & $900 \pm 100$ \\
\hline
\end{tabular}

All determinations were carried out at least three times .

*Polyphenolics are expressed as the mean \pm SD in $\mathrm{mM}$ of catechin equivalents per liter of beverage.

${ }^{* *}$ Total antioxidant activities are expressed as the mean \pm SD in $\mathrm{mM}$ of Trolox equivalents per liter of beverage.

Table 3. The polyphenolic contents and total antioxidant activities of 14 beverages using Folin assay and TEAC assay, respectively. 


\begin{tabular}{|c|c|c|c|}
\hline Name of plants & $\begin{array}{l}\text { TEAC Assay } \\
\text { TE mM/kg.dw }\end{array}$ & $\begin{array}{c}50 \% \text { Hemolysis* } \\
\mathrm{T}_{50} \text { min. }\end{array}$ & $\begin{array}{l}\text { Heinz body** } \\
\% \text { inhibition }\end{array}$ \\
\hline 1. Quercus infectoria & 9,490 & $>210$ & 100 \\
\hline 2. Areca catechu Linn. & 5,771 & $>210$ & 100 \\
\hline 3. $\quad$ Terminalia spp. & 5,216 & $>210$ & 100 \\
\hline 4. $\quad$ Phyllanthus emblica Linn. & 2,288 & $>210$ & 100 \\
\hline 5. $\quad$ Punica granutum Linn. & 2,157 & $>210$ & 100 \\
\hline 6. Camellia sinensis (green tea) & 2,906 & $>210$ & 100 \\
\hline 7. $\quad$ Eugenia caryophyllus & 1,400 & $>210$ & 100 \\
\hline 8. Mangifera indica Linn & 1,118 & $>210$ & 100 \\
\hline 9. Camellia sinensis(Oolong tea) & 2,204 & $>210$ & 100 \\
\hline 10. Rosa domescena Mill. & 1,035 & $>210$ & 100 \\
\hline 11. Tagetes erecta Linn. & 1,129 & $>210$ & 100 \\
\hline 12. Psidium guajava & 1,121 & $>210$ & 100 \\
\hline 13. Syzygium gratum & 718 & $>210$ & 100 \\
\hline 14. Impormoca reptans Poir. & 751 & $>210$ & 0 \\
\hline 15. Nelumbo Mucifera Gaertn & 1,014 & $>210$ & 0 \\
\hline 16. Smilax corbulalia Kunth & 1,308 & $>210$ & 0 \\
\hline 17. Nelumbo Mucifera Gaertn & 714 & $>210$ & 0 \\
\hline 18. Tamarindus indica Linn. & 932 & 180 & 0 \\
\hline 19. Garcinia mangostana & 1,095 & $>210$ & 0 \\
\hline 20. Cinnamomum cassia & 686 & $>210$ & 0 \\
\hline 21. Mesua ferrea Linn. & 357 & $>210$ & 0 \\
\hline 22. Ginkgo biloba (leaf) & 652 & $>210$ & 0 \\
\hline 23. Aiiium ascalonicum Linn. & 357 & $>210$ & 0 \\
\hline 24. Azodirachta indica A Juss. & 467 & $>210$ & 0 \\
\hline 25. Phyllanthus urinaria Linn. & 330 & $>210$ & 0 \\
\hline 26. Polygonium odoratum Lour. & 380 & $>210$ & 0 \\
\hline 27. Leucaena leucocephala & 316 & $>210$ & 0 \\
\hline 28. Erythrina suberosa Roxb. & 165 & $>210$ & 0 \\
\hline 29. Citrus hystrix DC. & 254 & $>210$ & 0 \\
\hline 30. Pandanus amaryllifolius Roxb & 141 & $>210$ & 0 \\
\hline 31. Positive control & & $120-150$ & 0 \\
\hline 32. Negative control & & $>210$ & 100 \\
\hline
\end{tabular}

*T50 min. = the time required to achieve $50 \%$ hemolysis.

** Blood smear on glass slides and counted RBC with Heinz body inside/1,000 of RBC. The results were reported in \% inhibition of Heinz body formation.

Table 4.Total antioxidant activities of 30 plant-extracts with inhibition effect on hemolysis, $\mathrm{T}_{50} \mathrm{~min}$, and inhibition effect on Heinz body formation. 


\section{Conclusion}

For our knowledge this current work is the first report of phenolic contents and total antioxidant activities of Thai herb. The value of antioxidant activities of plant polyphenols showed varied widely with the various types of herb. The range of antioxidant activities and total phenolic contents were several hundred-fold for herbal extracts and 15.6-fold using TEAC analysis and 6.5-fold using Folin assay for 33 brands of tea. Nevertheless there was a very good relationship between the results from the TEAC assay which can measure the antioxidants activities and polyphenols in herbal extracts $(r=0.988)$ and in tea extracts $(r=0.902)$. It is suggested that the process of oxidation to make black tea and oolong tea may cause decreasing their antioxidant activities, and the correlation between the antioxidant activities and total phenolic contents of tea extracts was lower than that of herbal extracts.

Tea is one of the most popular and widely consumed as daily beverage in the world. At present, tea has become an important agricultural product that more than 40 countries in the world, especially Thailand, grow tea trees within Asian countries producing $90 \%$ of the world output. All tea trees have their origin directly or indirectly in China and come from the same plant, Camellia sinensis. The composition of tea varies with the age of the leaf (plunking position), climate, horticultural practices and the process of storage (Lin et al., 1996, 1998). The different types of tea result from variation in processing of the leaves after they are harvested. The difference in processing results in different types of polyphenolics profiles between oxidation and no oxidation tea. There are different methods in manufacturing tea that give green tea (no oxidation) contains catechins. (Lin et al, 1998) black tea (fully oxidation tea) contains the polymeric compounds, thearubigins and theaflavins, and oolong tea (semi-oxidation tea) contains a mixture of the monomeric polyphenols and higher molecular weight theaflavins (Graham, 1992). Several epidemiological studies have shown beneficial effects of tea in cancer, cardiovascular, and neurological diseases (Zaveri, 2006).

Selected 30 types of herb with various polyphenol levels to study antioxidant activities by the modified methods which were developed by the author. For the test of AAPH induced hemolysis, most studies used isolated erythrocyte suspension for the simplicity of the system and the data interpretation. However fresh whole blood was employed as the ex vivo sample for this study to ensure the erythrocytes were tested in the least modified state. To study the effect of herbal extracts against oxidative damage to lipids and proteins in human erythrocytes, free radical initiators, AAPH and APHZ, were employed for inducing oxidative stress. AAPH was source of free radical formation capable of inducing oxidation of lipid and protein structurally located on erythrocyte membranes (Chaudhuri et al., 2007). The results showed that erythrocytes were more resistant to AAPH-induced oxidation when herbal extracts were added, most of them had very strong inhibition effect. It was showed that the $\mathrm{T}_{50}$ mins were longer than 3 and a half hours. Plant polyphenols are membrane-active antioxidant agents (Saija et al., 1995) and are the cell metabolism regulators by modulating the fluidity of lipid bilayer, since they have been demonstrated to control cell signal pathways by targeting receptors on the cell surface or by intercalating the lipid bilayer of membranes (Tarahovsky et al., 2008). Polyphenols 
interact with the surface of bilayer through hydrogen bonding have been shown to reduce the accessibility of oxidants, thus protecting the structure and function of membranes (Oteiza et al., 2005).

APHZ was a source of free radical formation inside cytosol of erythrocytes leading to induction of proteins mostly hemoglobin and lipid bilayer of membranes oxidation (Sangkitikomol et al., 2001). Phenylhydrazine in the presence of oxidase or peroxidase , it reacts with oxyhemoglobin to form phenylhydrazine radicals. Phenylhydrazine slowly oxidizes in aqueous solution to form $\mathrm{O}_{2} \bullet$ and $\mathrm{H}_{2} \mathrm{O}_{2}$ and the end products of the reaction are benzene and $\mathrm{N}_{2}$. Phenylhydrazine radical is the most damaging agent that can denature hemoglobin molecules. The oxidation of reactive sulfhydryl $(\mathrm{S}-\mathrm{H})$ groups creates disulfide bonds that may change the conformation of globin chains, resulting in precipitation of the hemoglobin molecules called Heinz bodies, then follows by membrane lipids oxidation and causing hemolysis (Winterbourn, 1985). Bioavailability differs greatly from one polyphenol to the other; therefore the most abundant polyphenols in plants are not leading to the highest concentrations of active metabolites in target cells. Gallic acid and isoflavones are the most absorbed polyphenols, followed by catechins, flavanones, and quercetin glucosides. The least absorbed polyphenols are proanthocyanidins, galloylated tea catechins, and anthocyanins (Manach et al., 2005). The results showed that some herbal extracts could inhibit Heinz body formation inside erythrocytes, but the other could not. This finding suggested that the process of proteins and lipids oxidation was still taking place as a result of insufficiency of absorbed polyphenols inside erythrocytes to inhibit oxidation.

The advantage of using erythrocytes (human living cells) as the models for screening test of antioxidant properties of herbal extracts, because it is simple and low cost to perform the analysis and the given data could be extrapolated to happen in human body.

\section{Acknowledgment}

This work was supported by Faculty of Allied Health Sciences, Sciences, Chulalongkorn University, Bangkok.

\section{References}

Ames, B.; Shigena, M. \& Hagen, T. (1993). Oxidants, antioxidants and the degenerative diseases of aging, Proceedings of the National Academic Sciences of the United State of America, Vol. 90, pp. 7915-7922, ISSN 0027-8424

Beecher, G. (2003). Overview of dietary flavonoids: nomenclature, occurrence and intake, Journal of Nutrition, Vol.133, pp. 3248-3254, ISSN 0022-3166

Bellezza, I.; Mierla,A. \& Minelli, A. (2010). Nrf2 and NF-KB and their concerted modulation in cancer pathogenesis and progression, Cancers,Vol. 2, pp. 483-497, ISSN 2072-6694

Chaudhary, A. \& Willett, K. (2006). Inhibition of human cytochrome CYP1 enzymes by flavonoids of St. John's wort, Toxicology, Vol. 217, pp. 194-205, ISSN 0300-483X.

Chaudhuri, S.; Banerjee, A.; Basu, K.; Sengupta, B. \& Sengupta, P. (2007). Interaction of flavonoids with red blood cell membrane lipids and proteins: Antioxidant and 
antihemolytic effects, International Journal of Biological Macromolecules Vol. 41, pp 4248, ISSN 0141-8130

Chen, H. \& Yen G. (1997). Possible mechanisms of antimutagen by various teas as judged by their effect on mutagenesis by 2-amino-3-methylmidazo[4,5-f]quinoline and benzo(a)pyrine, Mutation Research, Vol.393, pp. 115-122, ISSN 0921-8262

Cook, N. \& Samma, S. (1996). Flavonoids-chemistry, metabolism, cardioprotective effects and dietary sources, Journal of Nutritional Biochemistry, Vol. 7, pp. 66-76, ISSN 09552863

Graham, H. (1992). Green tea composition, consumption, and polyphenol chemistry, Preventive Medicine, Vol. 21, pp 334-350, ISSN 0091-7435

Greten, F. \& Karin, M. (2004). The IKK/NF-kappaB activation pathway-a target for prevention and treatment of cancer, Cancer Letters, Vol.206, pp. 193-199, ISSN 0304-3835

Guengerich, F. (2008). Cytochrome P450 and Chemical Toxicology. Chemical Research in Toxicology, Vol.21, pp. 70-83, ISSN 0893-228X

Halliwell, B. \& Gutteridge J. (2001) Free radicals in biology and medicine, Oxford Science Publication. Printed by Thomson Press (India) Ltd., pp.617-854, ISBN 0198500449 (Hbk), ISBN 0198500440 (Pbk)

Hayes, J. \& McMahon M. (2001). Molecular basis for the contribution of the antioxidant responsive element to cancer chemoprevention, Cancer Letters, Vol.174, pp. 103-113, ISSN: 0304-3835

Hertog, M.; Kromhout, D.; Aravanis, C.; Blackburn, H.; Buzina, R., et al. (1995). Flavonoid intake and long-term risk of coronary heart disease and cancer in the seven countries study, The Archives of Internal Medicine, Vol.155, pp. 381-386, ISSN 00039926

Keli, S.; Hertog, M.; Feskens, E. \& Kromhout, D. (1996). Dietary flavonoids, antioxidant vitamins, and incidence of stroke: the Zutphen study, The Archives of Internal Medicine, Vol. 156, pp.637-42, ISSN 0003-9926

Knekt, P.; Järvinen, R.; Seppänen, R.; Helibvaara, M.; Teppo, L.; et al. (1997). Dietary flavonoids and the risk of lung cancer and other malignant neoplasms, American Journal of Epidemiology, Vol.146, pp. 223-30, ISSN 0002-9262

Knekt, P.; Kumpulainen, J.; Järvinen, R.; Rissanen, H.; Heliövaara, M.; et al. (2002). Flavonoid intake and risk of chronic diseases, American Journal of Clinical Nutrition, Vol. 76, pp. 560-568, ISSN

Kohlmeier, L.; Weterings, K.; Steck, S. \& Kok F. (1997). Tea and cancer prevention: an evaluation of the epidemiological literature, Nutrition and Cancer, Vol.27, pp.1-13, ISSN 0163-5581

Lambert, J.; Hong, J.; Yang, G.; Liao, J. \& Yang C. (2005). Inhibition of carcinogenesis by polyphenols: evidence from laboratory investigations, American Journal of Clinical Nutrition, Vol.81, pp. 284S-291S, ISSN 0002-9165

Langley-Evans, S. (2001). Tea as a protective agent in cardiovascular health, Nutrition $\mathcal{E}$ Food Science, Vol. 31, pp. 75-78, ISSN 0034-6659

Lee, J. \& Surh Y. (2005). Nrf2 as a novel molecular target for chemopreventio, Cancer Letters, Vol. 224, pp. 171-184, ISSN 0304-3835 
Lin, Y.; Juan, I.; Chen, Y.; Liang, Y. \& Lin, J. (1996). Composition of polyphenols in fresh tea leaves and association of their oxygen-radical absorbing capacity with antiproliferative action in fibroblast cells, Journal of Agricultural and Food Chemistry, Vol. 44, pp1387-1394, ISSN 0021-8561

Lin, J.; Lin, C.; Liang, Y.; Lin-Shiau, S. \& Juan, I. (1998). Survey of catechins, galic acid and methylxanthines in green, oolong, pu-erh and black teas. Journal of Agricultural and Food Chemistry, Vol.46, pp3635-3642, ISSN 0021-8561

Lin, J. \& Liang, Y. (2000). Cancer chemoprevention by tea polyphenols, Proceedings of the National Science Council, Republic of China Part B, Vol.24, pp 1-13, ISSN 0255-6596

Ling, W.; Wang, L. \& Ma, J. (2002). Supplementation of the black rice outer layer fraction to rabbits decreases atherosclerotic plaque formation and increases antioxidant status, Journal of Nutrition, Vol. 132, pp. 20-26, ISSN 0022-3166

Manach, C.; Williamson, G.; Morand, C.; Scalbert, A. \& Rémésy, C. (2005). Bioavailability and bioefficacy of polyphenols in humans. I. Review of 97 bioavailability studies. American Journal of Clinical Nutrition, Vol. 81(suppl), pp 230S-242S, ISBN 0943685443

Nakamura, H. (1997). Effect of tea flavonoid supplimentation on the susceptibility of low density lipoprotein to oxidative modification, American Journal of Clinical Nutrition, Vol.66, pp. 261-266, ISSN 0002-9165

Nguyen, T.; Sherratt, P. \& Pickett C. (2003). Regulatory mechanisms controlling gene expression mediated by the antioxidant response element, Annual Review of Pharmacology and Toxicology, Vol. 43, pp. 233-60, ISSN 0362-1642

Oteiza, P.; Erlejman, A.; Verstraeten, S.; Keen, C. \& Fraga, C. (2005). Flavonoid-membrane interactions: A protective role of flavonoids at the membrane surface? Clinical and Developmental Immunology, Vol. 12, pp19-25, ISSN 1740-2522

Packer, L. \& Colman, C. (1999). The Antioxidant Miracle. Published by John Wiley and Sons, Inc. Canada, pp.1-248, ISBN 0-471-29768-2

Peluso, M. (2006). Flavonoids attenuate cardiovascular disease, inhibit phosphodiesterase, and modulate lipid homeostasis in adipose tissue and liver, Experimental Biology and Medicine, Vol.231, pp. 1287-1299, ISSN 1535-3702

Ramassamy, C. (2006). Emerging role of polyphenolic compounds in the treatment of neurodegenerative diseases: A review of their intracellular targets, European Journal of Pharmacology, Vol. 545, pp. 51-64, ISSN 0014-2999

Re, R.; Pellegrini, N.; Proteggente, A.; Pannala, A.; Yang, M. \& Rice-Evans, C. (1999). Antioxidant activity applying an improved ABTS radical cation decolerization assay, Free Radical in Biology and Medecine, Vol.26, pp.1231-1237, ISSN 08915849

Saija, A.; Scalese, M.; Lanza, M. \& et al. (1995). Flavonoids as antioxidant agents: importance of their interaction with biomembranes, Free Radical in Biology and Medecine, Vol.19, pp481-486, ISSN 0891-5849

Sangkitikomol, W.; Krikreangsak, N. \& Klaisiri, A. (2001). The preventive effect of antioxidants in vegetables and Thai herbs on Heinz bodies formation in red blood cells induced by oxidant agent:Acetylphenylhydrazine, Journal of Allied Health Sciences, Vol.1, pp. 46-54, ISBN 1513-4865, Bangkok, Thailand 
Sangkitikomol, W.; Tencomnao, T. \& Rocejanasaroj, A. (2010a). Antioxidant effects of anthocyanins-rich extract from black sticky rice on human erythrocytes and mononuclear leukocytes, African Journal of Biotechnology, Vol. 9, pp. 8222-8229, ISSN 1684-5315

Sangkitikomol, W.; Tencomnao, T. \& Rocejanasaroj, A. (2010 b). Effects of Thai black sticky rice extract on oxidative stress and lipid metabolism gene expression in HepG2 cells, Genetics and Molecular Research,Vol. 9, pp. 2086-2095, ISSN1676 -56

Sen, S.; Chakraborty, R.; Sridhar, C.; Reddy, Y. \& De, B. (2010). Free radicals, antioxidants, diseases and phytomedicines: current status and future prospect, International Journal of Pharmaceutical Sciences Review and Research, Vol. 3, pp. 91-100, ISSN 0976044X

Singleton, V.; Orthofer, R. \& Lamuela-Raventos R. (1999). Analysis of total phenols and other oxidation substrates and antioxidants by means of Folin-Ciocalteu reagent. In Method in Enzymology. Packer L.(ed). Academic Press, Orlando FL. USA, Vol. 299, pp. 152-178, ISBN 0-12-182236-2

Steinmetz, K. \& Potter J. (1991). Vegetables, fruit, and cancer. II. Mechanisms, Cancer Causes and Control, Vol.2, pp. 427-42, ISSN 0957-5243

Surh, Y. (2003). Cancer chemoprevention with dietary phytochemicals. Nature Reviews Cancer, Vol. 3, pp. 768-80, ISSN 1474-175X

Tarahovsky, Y.; Muzafarov, E. \& Kim, Y. (2008). Rafts making and rafts braking: how plant flavonoids may control membrane heterogeneity. Molecular and Cellular Biochemistry Vol. 314, pp. 65-71, ISSN 0300

Thomasset, S.; Berry, D.; Garcea, G.; Marczylo, T.; Steward, W. \& Gescher A. (2006). Dietary polyphenolic phytochemicals-Promising cancer chemopreventive agents in humans? A review of their clinical properties, International Journal of Cancer, Vol. 120, pp.451-458, ISSN 0020-7136

Tsuji, PA. \& Walle, T. (2007). Benzo[a]pyrene-induced cytochrome P450 1A and DNA binding in cultured trout hepatocytes - Inhibition by plant polyphenols, ChemicoBiological Interactions, Vol. 169, pp. 25-31, ISSN 0009-2797

Virgili, F. \& Marino, M. (2008). Regulation of cellular signals from nutritional molecules: a specific role for phytochemicals, beyond antioxidant activity, Free Radical in Biology and Medecine, Vol. 45, pp.1205-1216, ISSN 0891-5849

Wang, Q.; Han, P.; Zhang, M.; Xia, M.; Zhu, H.; et al. (2007). Supplementation of black rice pigment fraction improves antioxidant and anti-inflammatory status in patients with coronary heart disease, Asia Pacific Journal of Clinical Nutrition, Vol.16(Suppl 1), pp. 295-301, ISSN 0964-7058

Winterbourn, C. (1985). Free-radical production and oxidative reactions of hemoglobin. Environmental Health Perspectives, Vol.64, pp. 321-330, ISSN 0091-6765

Zaveri, T. (2006). Green tea and its polyphenolic catechins: Medicinal uses in cancer and noncancer applications. Life Sciences, Vol.78, pp. 2073-2080, ISSN 0024-3205

Zhang, Y. \& Gordon, B. (2004). A strategy for cancer prevention: stimulation of the Nrf2ARE signaling pathway, Molecular Cancer Therapeutics, Vol. 3, pp. 885-893, ISSN 1535-7163 
Ziegler, R. (1991). Vegetables, fruits and carotenoids and risk of cancer, American Journal of Clinical Nutrition, Vol. 53(suppl.), pp. 251s-259s, ISSN 0002-9165 


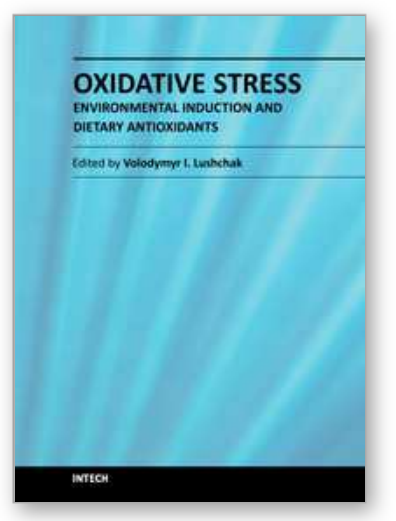

\section{Oxidative Stress - Environmental Induction and Dietary Antioxidants}

Edited by Dr. Volodymyr Lushchak

ISBN 978-953-51-0553-4

Hard cover, 388 pages

Publisher InTech

Published online 02, May, 2012

Published in print edition May, 2012

This book focuses on the numerous applications of oxidative stress theory in effects of environmental factors on biological systems. The topics reviewed cover induction of oxidative stress by physical, chemical, and biological factors in humans, animals, plants and fungi. The physical factors include temperature, light and exercise. Chemical induction is related to metal ions and pesticides, whereas the biological one highlights hostpathogen interaction and stress effects on secretory systems. Antioxidants, represented by a large range of individual compounds and their mixtures of natural origin and those chemically synthesized to prevent or fix negative effects of reactive species are also described in the book. This volume will be a useful source of information on induction and effects of oxidative stress on living organisms for graduate and postgraduate students, researchers, physicians, and environmentalists.

\section{How to reference}

In order to correctly reference this scholarly work, feel free to copy and paste the following:

Warin Sangkitikomol (2012). Antioxidants in Thai Herb, Vegetable and Fruit Inhibit Hemolysis and Heinz Body Formation in Human Erythrocytes, Oxidative Stress - Environmental Induction and Dietary Antioxidants, Dr. Volodymyr Lushchak (Ed.), ISBN: 978-953-51-0553-4, InTech, Available from: http://www.intechopen.com/books/oxidative-stress-environmental-induction-and-dietaryantioxidants/antioxidants-in-thai-herb-vegetable-and-fruit-inhibit-hemolysis-and-heinz-body-formation-inhuman-e

\section{INTECH}

open science | open minds

\section{InTech Europe}

University Campus STeP Ri

Slavka Krautzeka 83/A

51000 Rijeka, Croatia

Phone: +385 (51) 770447

Fax: +385 (51) 686166

www.intechopen.com

\section{InTech China}

Unit 405, Office Block, Hotel Equatorial Shanghai

No.65, Yan An Road (West), Shanghai, 200040, China

中国上海市延安西路65号上海国际贵都大饭店办公楼 405 单元

Phone: +86-21-62489820

Fax: $+86-21-62489821$ 
(C) 2012 The Author(s). Licensee IntechOpen. This is an open access article distributed under the terms of the Creative Commons Attribution 3.0 License, which permits unrestricted use, distribution, and reproduction in any medium, provided the original work is properly cited. 